\title{
Using video to learn practical techniques in physiotherapy education
}

\author{
M Rowe, PhD, MSc, BSc (Physiotherapy); B Sauls, MSc, BSc (Physiotherapy) \\ Department of Physiotherapy, Faculty of Community and Health Sciences, University of the Western Cape, Cape Town, South Africa
}

Corresponding author: M Rowe (mrowe@uwc.ac.za)

\section{Problem}

The traditional approach to developing practical skills in physiotherapy education presents several challenges. Because of larger class sizes, it is difficult for all students to see clearly what is being demonstrated, and because of limited staff numbers, lecturers cannot necessarily spend enough time with students to observe and correct their techniques. ${ }^{[1]}$ This study aimed to explore an alternative means of instruction that is emerging as an area of interest in physiotherapy education. Video-based learning is increasingly being used in programmes with high levels of practical content to free up time in the classroom for lecturers to help students to problem solve instead. Video-based learning allows students to learn a practical technique using only online resources for instruction and demonstration components.

The project received ethical clearance from the University of the Western Cape Research Committee (ref. no. HS/16/5/7).

\section{Approach}

A total of 41 students chose to participate in the study. They were randomly divided into the video-based learning group $(n=21)$ and the face-to-face group $(n=20)$. Both groups learnt the static and dynamic patellar apprehension tests in a session of 20 minutes, working in groups of 3 - 5 students. The face-to-face group observed the lecturer demonstrate the technique and then practised in groups. The video-based learning group watched both techniques by video, which was supplemented with images and text descriptions on a single web page. These students also practised in groups, but there were no lecturers present to clarify any questions they might have had at the time. Two weeks later, both groups of students were tested using an objective structured practical examination (OSPE). The lecturer designed the OSPE and the researchers marked the students' performance of both techniques. As the study was voluntary, students could choose whether to take the OSPE. While all 21 students in the video-based group took the test, only 9 of the 20 in the face-to-face group were assessed.

\section{Outcome}

The study found that the abovementioned two practical techniques could be taught using only a video-based teaching session, as $76 \%(n=16 / 21)$ of the students in the video-based group passed the OSPE, compared with
$55 \%(n=5 / 9)$ of those in the face-to-face group. The average grades of the video-based learning group (67\%) were similar to those of the face-to-face learning group (63\%). We acknowledge that using performance scores in the assessment is a poor indicator of the success of the intervention and that these findings should be interpreted with caution. However, the aim of this short communication is not to produce generalisable findings, but rather to describe a novel intervention in physiotherapy education that might serve as a foundation for more rigorous feasibility studies.

In a face-to-face teaching session, much of the time may be spent on the mechanics of the practical technique, meaning that there may be limited time to engage with the types of questions that emerge after practice and that improve students' understanding and application of the technique. ${ }^{[2]}$ This small pilot study suggests that some practical physiotherapy techniques might be taught using only video-based learning, including techniques that are relatively simple to describe and model. Using video-based approaches might help lecturers free up additional time in class for discussion, increased engagement and problem solving around more advanced techniques.

Declaration. The claims made in this article represent the perspectives of the authors and not of the institution and employer.

Acknowledgements. The authors would like to acknowledge the contributions of the following undergraduate students: $\mathrm{C}$ van der Merwe, A Austin, C Austin, L Alberts and A Karsten, who conducted the research and analysed the data.

Author contributions. MR conceptualised the study and prepared the proposal for ethical approval; BS prepared the first draft of the manuscript and assisted with the revision of the manuscript; and both authors were involved with the preparation of the manuscript and gave final approval for submission.

Funding. None.

Conflicts of interest. None.

\footnotetext{
1. George A, Blaauw D, Green-Thompson L, et al. Comparison of video demonstrations and bedside tutorials for teaching paediatric clinical skills to large groups of medical students in resource-constrained settings. Int Educational Technol High Educ 2019;16(34):1-16. https://doi.org/10.1186/s41239-019-0164-z

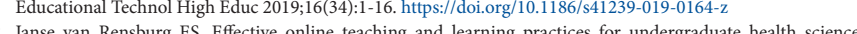
2. Janse van Rensburg ES. Effective online teaching and learning practices for undergraduate health scie
students: An integrative review. Int I Afr Nurs Sci 2018:9:73-90. https://doi.org/10.1016/.ijans.2018.08.004
}

Accepted 19 November 2019

Afr J Health Professions Educ 2020;12(2):52. https://doi.org/10.7196/AJHPE.2020.v12i2.1271 